\title{
Imatinib mesylate decreases the cytotoxic effect of roscovitine on human glioblastoma cells in vitro and the role of midkine
}

\author{
MINE ERGUVEN $^{1}$, AYHAN BILIR ${ }^{2}$, NURAY YAZIHAN ${ }^{3}$, SEVAL KORKMAZ $^{4}$, \\ ESIN AKTAS $^{5}$, CEM OVALIOGLU ${ }^{6}$, TOLGA DUNDAR ${ }^{7}$ and HAKAN SEYITHANOGLU ${ }^{7}$ \\ ${ }^{1}$ Faculty of Medicine, Department of Biochemistry, Yeni Yüzyıl University; \\ ${ }^{2}$ Department of Histology and Embryology, Istanbul Faculty of Medicine, Istanbul University, Istanbul; \\ ${ }^{3}$ Faculty of Medicine, Department of Pathophysiology, Ankara University, Ankara; \\ ${ }^{4}$ Cell Culture and In Vitro Screening Laboratory, Abdi İbrahim Pharmaceuticals R\&D Center; \\ ${ }^{5}$ Department of Immunology, Institute of Experimental Medicine (DETAE), Istanbul University; \\ ${ }^{6}$ Department of Neurosurgery, Bakırkoy Hospital; ${ }^{7}$ Department of Neurosurgery, \\ Faculty of Medicine, Bezmialem Vakıf University, Istanbul, Turkey
}

Received June 7, 2011; Accepted August 31, 2011

DOI: $10.3892 / \mathrm{ol} .2011 .434$

\begin{abstract}
The purpose of the present study was to overcome resistance to imatinib (IM) by combining it with roscovitine (ROSC) and to investigate whether or not midkine (MK) had an effect on this combination in the treatment of glioblastoma (GBL). Human T98 GBL cells were used to evaluate the effects of IM $(10 \mu \mathrm{M})$, ROSC $(200 \mu \mathrm{M})$ and their combination on the cell proliferation index, apoptotic index, the apoptotic protein and anti-apoptotic protein levels, and ultrastructure. All applications decreased the cell proliferation index and increased the apoptotic index, but ROSC was the most efficient drug and the second most efficient drug was IM. Notably, ROSC increased anti-apoptotic proteins levels (PDGFR- $\alpha$, AQP-4, hTERT), COX-1 activity and ribosome numbers. The effects of ROSC on hTERT, MK, AQP-4 and MRP-1 levels and COX-1 activity were reported for the first time. ROSC induced the highest increase in caspase-3 levels. Autophagy was not involved in the activity of ROSC in GBL spheroids. The combination of IM with ROSC showed an antagonist effect in the treatment of human GBL cells. The combination group decreased certain anti-apoptotic protein levels (PDGFR- $\alpha$, EGFR, p-gp, MRP-1 and MK), cAMP levels, COX-1 activity and apoptotic protein levels (caspase-3). However, it induced the highest increase in hTERT levels and COX-2 activity. Ribosome numbers were much lower than those in the ROSC group and no autophagic vacuole was observed. In conclusion, more investigations are required to identify the key regulatory components that are
\end{abstract}

Correspondence to: Dr Mine Erguven, Yeni Yüzyıl University, Faculty of Medicine Department of Clinical Biochemistry, Yilanli Ayazma Caddesi No. 26, 34093 Cevizlibag-Topkapi, Istanbul, Turkey E-mail: mine.erguven@gmail.com

Key words: imatinib mesylate, roscovitine, glioblastoma, midkine, combination chemotherapy responsible for this antagonism; however, the determination of this combination therapy as a failure therapy may be precautionary for oncologists in the treatment of GBL patients and potentially may contribute to the efficacy of new therapeutic regimens.

\section{Introduction}

Cyclins and cyclin-dependent kinases (CDK) are the regulatory proteins of the cell cycle. Uncontrolled growth and proliferation of cancer cells are the result of their over activity. R-roscovitine (ROSC) (also known as CYC202 and Seliciclib) is a second-generation CDK inhibitor reducing tumor growth and proliferation, inducing cell death by competing for ATP binding sites on these CDKs. ROSC has inhibitory effects on cyclin E/CDK2, cyclin H/CDK7 and cyclin T/CDK9. In addition to their cell cycle regulatory function, CDK7 and CDK9 play significant roles in the regulation of RNA polymerase II-mediated transcription $(1,2)$. ROSC induces growth arrest and apoptosis through activation of the p53 gene, inhibition of ribosome biogenesis at an early rRNA processing level and blockage of the RNA polymerase II-dependent transcription, inhibition of mitogen-activated kinases (MAPKs) and pyridoxal kinase, reduction of anti-apoptotic proteins, such as survivin, p-glycoprotein (p-gp), nuclear factor $\kappa$-light-chainenhancer of activated $\mathrm{B}$ cells $(\mathrm{NF}-\kappa \mathrm{B})$, vascular endothelial growth factor (VEGF) and inhibition of potassium channels named Human ether-a-go-go-related gene (HERG) (3-10). Besides ongoing in vitro and in vivo experiments regarding its anti-neoplastic effects, ROSC has entered phase II clinical trials as a treatment for various tumors such as non-small cell lung cancer and advanced solid tumors (1).

Tyrosine kinase receptors (TKRs) play significant roles in tumor progression and therapy resistance. Activation of these tyrosine kinases (TKs) causes a cascade reaction, ultimately leading to DNA synthesis and cell division (10). Small molecule inhibitors were designed to block the enzymatic 
function of the TKs. A prototype tyrosine kinase inhibitor (TKI), imatinib mesylate (IM), known as Gleevec, was the first to be introduced into clinical oncology for leukemia, and was followed by other TKI drugs such as gefitinib, erlotinib, sorafenib, sunitinib and dasatinib. TKIs share the same mechanism of action as the competitive ATP inhibition at the catalytic binding site of TK; however, their TK targets are different $(11,12)$. IM inhibits Abelson cytoplasmic tyrosine kinase $(\mathrm{ABL}), \mathrm{c}-\mathrm{Kit}$, the platelet-derived growth factor receptor (PDGFR) and epidermal growth factor receptor (EGFR) (12,13). In addition, IM showed its toxicity by inducing mitochondrial damage (14).

Glioblastoma (GBL) is the most common primary malignant intraparenchymal brain tumor and accounts for the majority of diagnoses. Prognosis of GBL remains poor and GBL is accepted as virtually incurable due to its marked heterogeneity, which leads to resistance to various radiation and/or chemotherapy modalities (15). Reports showed that IM had anti-neoplastic effects on GBL; however, drug efflux proteins, particularly p-gp, decreased its efficiency primarily, and mutations at the TKR secondarily (16-18). In the present study, our aim was to overcome resistance to IM through its combination with ROSC, due to the inhibitory activities of the latter on $\mathrm{p}$-gp and downstream signaling of TKR as CDKs. In addition, we also investigated whether MK, a newly discovered resistance factor, had an effect on this combination.

Briefly, a heparin-binding growth factor, MK, was originally reported to be the product of a retinoic acid-responsive gene during embryogenesis (19). MK expression is high during embryogenesis; however, MK is undetectable in healthy adults and only reappears in the body as a part of disease pathogenesis. High frequency and massive expression in advanced tumors has been detected (20-22). High levels of MK expression correlate with the progression of human astrocytomas: MK mRNA and protein expression levels were higher in high-grade astrocytomas (anaplastic astrocytomas and GBLs) than in low-grade astrocytomas (23). Consequently, besides the well-known survival and resistance factors, the investigation of the effect of MK on the activity of ROSC and the new combination model with IM may give information about the reasons for success or failure of treatment.

\section{Materials and methods}

Monolayer and spheroid cell cultures. The T98G GBL cell line was supplied by the American Type Culture Collection (ATCC; Rockville, USA) and was grown in a monolayer culture in Dulbecco's modified eagle's medium-F12 (DMEMF12; Biological Industries, Israel) supplemented with $10 \%$ heat-inactivated fetal calf serum, $1 \mathrm{mM}$ sodium pyruvate, $0.1 \mathrm{mM}$ non-essential aminoacid solution, $50 \mathrm{U} / \mathrm{ml}$ penicillin and streptomycin (Sigma Chemical Co., St. Louis, MO, USA). Cells in semi-confluent flasks were harvested using $0.05 \%$ trypsin and $0.53 \mathrm{mM}$ EDTA solution (Sigma Chemical Co.) and centrifuged following the addition of DMEM-F12 for trypsin inactivation and then resuspended in culture medium. Following the trypan blue exclusion assay, GBM cells were plated in six-well culture plates containing $5 \mathrm{ml}$ DMEM-F12 medium at a concentration of $5 \times 10^{5}$ cells/well with $100 \%$ vitality. An in vitro multicellular T98G GBL spheroid model was established using a liquid overlay technique. Briefly, semiconfluent monolayer cell cultures were trypsinized and single cells with $100 \%$ vitality were cultured in 3\% Noble agarcoated (Difco, USA) six-well culture plates containing $5 \mathrm{ml}$ DMEM-F12 medium at a concentration of $1 \times 10^{6}$ cells/well.

Experimental design. IM and ROSC were applied at a volume of $100 \mu \mathrm{l}$ to monolayer cultures of T98G GBL cells in concentrations ranging from 1 to $200 \mu \mathrm{M}$, whereas the negative control cells received only nutrient medium alone. Cultures were incubated for $72 \mathrm{~h}$. Inhibition concentration 50 $\left(\mathrm{IC}_{50}\right)$ values were determined as $10 \mu \mathrm{M}$ for IM and $200 \mu \mathrm{M}$ for ROSC. Experiments were performed in monolayer and 3D cultures of human T98G GBL cells. Groups were determined as control, IM $(10 \mu \mathrm{M})$, ROSC $(200 \mu \mathrm{M})$ and their combination. For each experimental group $n=6$. Experiments were repeated three times and achieved similar results. Cell proliferation (total cell number), apoptotic cell death analysis by flow cytometric annexin-V-fluorescein isothiocyanate/propidium iodide (PI) (annexin-V-FITC/PI) staining, caspase-3, MK and EGFR levels by enzyme-linked immunosorbent assay (ELISA), protein levels of PDGFR- $\alpha$ multi-dug resistance protein-1 (MRP-1), p170, human telomerase reverse transcriptase (TERT) and aquaporin-4 (AQP-4) (Western blotting), cAMP levels (RIA: Radioimmunoassay), cyclooxygenase (COX) activity, morphology (SEM: Scanning Electron Microscopy) in monolayer cultures and cell ultrastructure (TEM: Transmission Electron Microscopy) in spheroid cultures were evaluated for $72 \mathrm{~h}$.

Cell proliferation and apoptotic indices. Cells were harvested every $24 \mathrm{~h}$ for $72 \mathrm{~h}$ and the total cell number was determined by using an automated cell counter (nucleocounter, Denmark). One of the manifestations of apoptosis is the translocation of phosphatidylserine (PS) from the inner membrane to the outer side of the plasma membrane. Externalization of PS was studied by the annexin-V-binding assay. Briefly, cells were washed twice with phosphate-buffered saline (PBS) and resuspended by binding buffer containing 0.01 M HEPES, $0.14 \mathrm{mM} \mathrm{NaCl}$ and $2.5 \mathrm{mM} \mathrm{CaCl}{ }_{2}$. A cell suspension $\left(1 \times 10^{5}\right.$ cells in $100 \mu \mathrm{l}$ ) in binding buffer was incubated with $5 \mu \mathrm{l}$ of FITC-labeled annexin V (BD Pharmingen, San Diego, CA, USA) dye, and PI for $15 \mathrm{~min}$ in the dark at room temperature. Following incubation, the PI fluorescence and annexin V were measured simultaneously in a BD FACS/Calibur and analyzed with the instrument's operating software (CellQuest, BD Pharmingen). Data acquisition and analysis were undertaken with CellQuest and WinMDI programs.

Caspase-3 levels. Cell culture supernatants were analyzed for caspase-3 levels in triplicate, using fluorimetric kits (Sigma Aldrich, MO, USA). The caspase-3 fluorimetric assay is based on the hydrolysis of the peptide substrate acetyl-Asp-GluVal-Asp-7-amido-4-methylcoumarin (Ac-DEVD-AMC) by caspase 3 , resulting in the release of the fluorescent 7-amino4-methylcoumarin (AMC) moiety. Cells $\left(1 \times 10^{4}\right)$ seeded in each well of 96-well plates were washed twice in PBS and incubated in CHAPS lysis buffer at $4^{\circ} \mathrm{C}$ for $20 \mathrm{~min}$. Cell lysate (5 $\mu \mathrm{l})$ was transferred into the wells of other 96-well plates, 
then incubated with $5 \mu \mathrm{l}$ of $2 \mathrm{mM}$ Ac-DEVD-pNA peptide substrate and $200 \mu \mathrm{l}$ of assay buffer (HEPES $20 \mathrm{mM}$, pH 7.4, CHAPS $0.1 \%$, DTT $5 \mathrm{mM}$, EDTA $2 \mathrm{mM}$ ) at $37^{\circ} \mathrm{C}$ for $1 \mathrm{~h}$ in an incubator. The concentration of AMC released was quantified by reading in a fluorometer with a $360 \mathrm{~nm}$ excitation filter and a $460 \mathrm{~nm}$ emission filter for optimal sensitivity.

Cell cycle. The effects of drugs on the cell cycle were examined using a DNA analysis kit (BD Pharmingen) according to the manufacturer's instructions. Briefly, T98G cells were induced at a cell density of $5 \times 10^{5}$ cells/ml in the presence of each drug applied separately and in combination for various time intervals (24 and 72 h). Cells were then harvested, centrifuged, washed and resuspended in buffer [dimethyl sulfoxide (DMSO) in sucrose-sodium citrate] for $5 \mathrm{~min}$ at room temperature. A mixture of trypsin in spermine tetrahydrochloride detergent buffer was added and samples were incubated for $20 \mathrm{~min}$ at room temperature. Following the addition of RNase A and trypsin inhibitor in spermine buffer, cells were incubated with PI, in darkness for $20 \mathrm{~min}$ at $4^{\circ} \mathrm{C}$. Flow cytometric analysis was performed immediately using a Facscan flow cytometer (FACS Diva, Becton-Dickinson, CA, USA) and fluorescence intensity data were acquired using the instrument's operating software (CellQuest, BD Pharmingen). The percentages of the analyzed cell population in G0/G1-, $\mathrm{S}$ - or G2/M-phases were determined by the Mod Fit cell-cycle analysis program.

MK levels. Cell culture supernatants were analyzed for MK levels in triplicate, using ELISA kits (PeproTech, NJ, USA). The lower detection limit of the assay was $150 \mathrm{pg} / \mathrm{ml}$ for MK. MK levels were measured by an ELISA system in which polyclonal anti-human MK was used as a capture antibody (Peprotech). Detection was by biotinylated polyclonal anti-human MK antibody (Peprotech) followed by streptavidin HRP (Sigma) and a TMB enzyme substrate system (Sigma). The reaction was stopped by $1 \mathrm{M} \mathrm{H}_{2} \mathrm{SO}_{4}$ and readings were made at $450 \mathrm{~nm}$ by a spectrometer (M2, Molecular devices, CA, USA).

cAMP levels. Following centrifugation, the supernatant was removed and $0.1 \mathrm{~N} \mathrm{HCl}$ with DMEM-F12 medium (1:1) was added to cells to stop the reaction at each $24 \mathrm{~h}$ interval. Briefly, $25 \mu \mathrm{l}$ of the samples were used to measure cAMP levels. cAMP accumulation was measured in the supernatants according to the method previously described, with some modifications (20). cAMP was determined by radio-immunoassay using the acetylation protocol. High-affinity rabbit anti-cAMP antibodies were generated in our laboratory using BSA-conjugated cAMP. Succinyl-cAMP tyrosine methylester (ScAMP-TME) was iodinated by the chloramine-T method. Mono- and diiodo ScAMP-TME were used as tracer ligands for the radioimmunoassay (RIA) and then purified by gel-filtration chromatography (Sephadex G 25 superfine), equilibrated and eluted with $1 \mathrm{M}$ sodium acetate ( $\mathrm{pH}$ 5.0).

EGFR levels. Cell culture supernatants were analyzed for EGFR levels in triplicate, using ELISA kits (SABiosciences, Germany). EGFR levels were measured by an ELISA system in which anti-human EGFR was used as capture antibody
(SABiosciences) and detection was by biotinylated polyclonal anti-human EGFR antibody (SABiosciences) followed by streptavidin HRP (SABiosciences) and a TMB enzyme substrate system (SABiosciences). The reaction was stopped by $2 \mathrm{M} \mathrm{H}_{2} \mathrm{SO}_{4}$ and readings were made at $450 \mathrm{~nm}$ by a spectrometer (M2, Molecular devices).

Cyclooxygenase activity. COX activity was indirectly measured by utilizing TMPD as a co-substrate with arachidonic acid. TMPD does not turn over without the presence of a hydroperoxide substrate. TMPD oxidation was monitored spectrophotometrically with a 96-well plate reader at $610 \mathrm{~nm}$ every $1 \mathrm{~min}$ for $10 \mathrm{~min}$. Enzyme $(100 \mu \mathrm{g} / \mathrm{ml})$ and inhibitor $(100 \mu \mathrm{g} / \mathrm{ml})$ were mixed for various amounts of time in $100 \mathrm{mM}$ Tris- $\mathrm{HCl}(\mathrm{pH} 8.0)$, containing $10 \mu \mathrm{M}$ heme and $3 \mu \mathrm{M}$ EDTA. This mixture was added into wells and incubated for $15 \mathrm{~min}$ at $25^{\circ} \mathrm{C}$. The reaction was then started by the addition of a mixture of $100 \mu \mathrm{M}$ arachidonic acid and $120 \mu \mathrm{M}$ TMPD.

Protein levels of PDGFR- $\alpha$, MRP-1, p170, hTERT and $A Q P-4$. Expression of PDGFR- $\alpha$, MRP-1, p170, hTERT and AQP-4 proteins were detected by Western blot analysis. Cells $\left(2 \times 10^{6}\right)$ were lysed for $15 \mathrm{~min}$ at $4^{\circ} \mathrm{C}$ in RIPA lysis buffer. Protein content was assessed using a BCA protein assay (Pierce, Rockford, IL, USA). Samples with equal amounts of protein were separated on a 7 and $10 \%$ sodium dodecyl sulfate polyacrylamide electrophoresis (SDS-PAGE) gel, transferred to polyvinylidene fluoride (PVDF) membranes and detected with PDGFR- $\alpha$, MRP-1, bcl-2, COX-2 (1/100 dilution, all from Santa Cruz Biotechnology, Santa Cruz, CA, USA) and $\beta$-actin (1/1000 dilution, Pierce) antibodies. Protein bands were visualized with the enhanced chemiluminescence (ECL) Advance Western blot detection reagents (GE Healthcare Life Sciences, NJ, USA) and quantified by the ImageJ image processing program (National Institutes of Health, Bethesda, MD, USA).

Ultrastructure. Harvested spheroids were fixed with $2.5 \%$ glutaraldehyde in $0.1 \mathrm{M}$ sodium cacodylate buffer and post-fixed in $1 \%$ osmium tetroxide in $0.1 \mathrm{M}$ sodium cacodylate buffer for $1 \mathrm{~h}$ at $4^{\circ} \mathrm{C}$. Cells were incubated in $1 \%$ uranyl acetate for $1 \mathrm{~h}$ at $4^{\circ} \mathrm{C}$, dehydrated in a graded acetone series and embedded in Epon 812. Samples were cut using a rotating blade microtome (Leica, Heerbrugg, Switzerland) and 70-nm sections were mounted on copper grids. Sections were subsequently stained with 5\% uranyl acetate and counterstained with Reynold's lead citrate. Sections were examined with a Jeol-Jem 1011 transmission electron microscope. Images were captured at a number of magnifications.

Statistical analysis and determination of synergism. SPPS 17.0 statistical software (SPSS, Inc., Chicago, IL, USA) was used for the statistical analysis. The results were statistically analyzed using the Student's t-test. Data were presented as the mean \pm SEM. $\mathrm{P}<0.05$ was considered to be statistically significant. Synergy was determined as previously described (24-26). Briefly, synergism was determined using the formula: combination index (CI): D1/(DX)1 + D2/(DX)2, where D1 is the tested concentration of IM used in combination with ROSC, D2 is the tested concentration of ROSC used in combination with IM, 


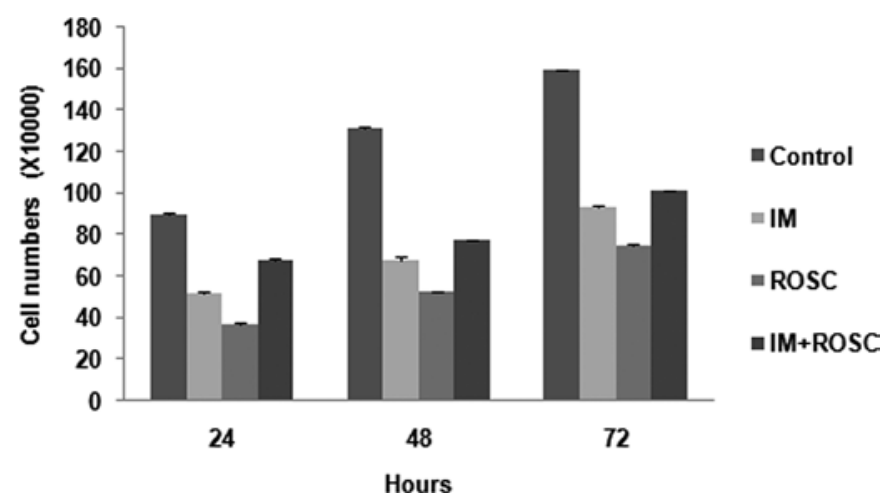

Figure 1. Cell proliferation index. IM, imatinib mesylate; ROSC, roscovitine; IM+ROSC, the combination of imatinib mesylate and rescovitine. Results are the mean of three different experiments and are presented as the mean \pm standard error mean (SEM).

(DX)1 is the concentration of a singly applied IM and (DX)2 is the concentration of a singly applied ROSC. A CI value of 1 indicates an additive effect, a CI value $<1$ indicates a synergistic effect and a CI value $>1$ indicates an antagonist effect.

\section{Results}

Cell proliferation. The effects of drug applications are shown in Fig. 1. The cell number of the control group showed a proportional increase for $72 \mathrm{~h}$. All drug treatments inhibit cell proliferation of the T98G GBL cell lines for $72 \mathrm{~h}(\mathrm{p}<0.05)$ in a time-dependent manner. The rank from highest decrease to lowest decrease was determined as the ROSC, IM and the combination groups, respectively $(\mathrm{p}<0.05)$.

Apoptotic index and caspase-3 levels. Fig. 2 clearly shows various levels of apoptosis (Fig. 2A) and caspase-3 activity (Fig. 2B) induced by these drug applications. ROSC, IM and the combination group increased apoptosis and caspase-3 activity for $72 \mathrm{~h}(\mathrm{p}<0.05)$. ROSC induced the highest apoptotic index, but the combination group led to the lowest apoptotic index $(\mathrm{p}<0.05)$.

Cell cycle. T98G GBL cells induced growth arrest in the S-phase of the cell cycle within $24 \mathrm{~h}$ of drug treatments $(\mathrm{p}<0.05)$. ROSC and the combination group increased the population in the $\mathrm{G} 2 / \mathrm{M}$ phases, but IM induced growth arrest in the G0+G1 at $72 \mathrm{~h}(\mathrm{p}<0.05)$ (Fig. 3).

MK levels. The alterations in MK levels for $72 \mathrm{~h}$ are shown in Fig. 4. All drugs alone and in combination decreased MK levels for $72 \mathrm{~h}$. The rank from highest decrease to lowest decrease was determined as the ROSC, IM and combination groups for $72 \mathrm{~h}$, respectively $(\mathrm{p}<0.05)$.

cAMP levels. All drug applications decreased cAMP levels $(\mathrm{p}<0.05)$. ROSC induced the highest decrease in cAMP levels, but the decrease by the combination group was similar to that
A

CONTROL

IM

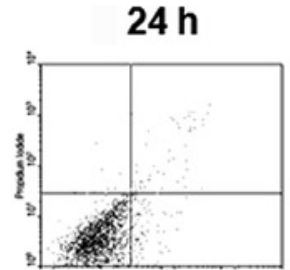

Rosc

IM+ROSC
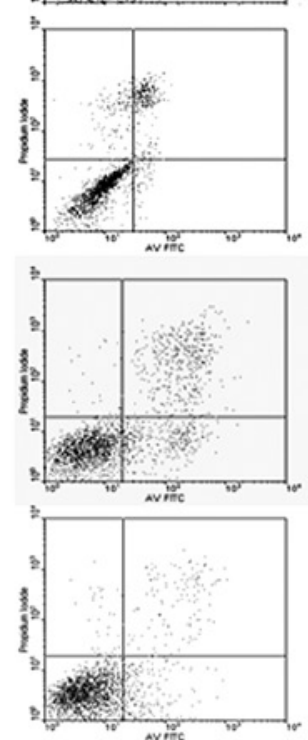

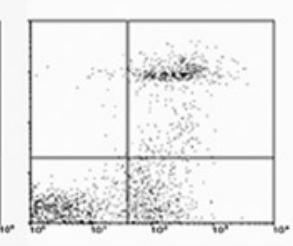

$72 \mathrm{~h}$
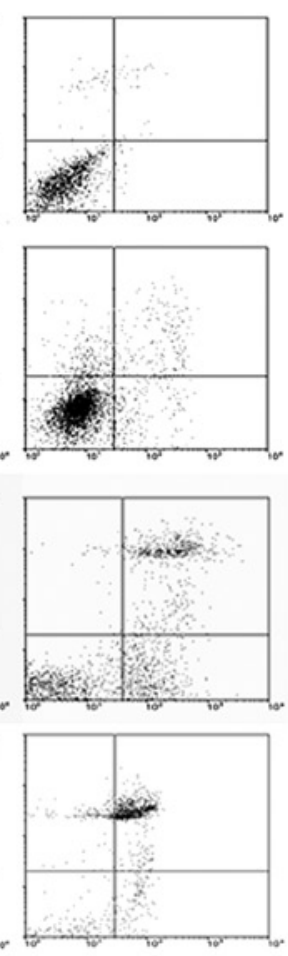

B
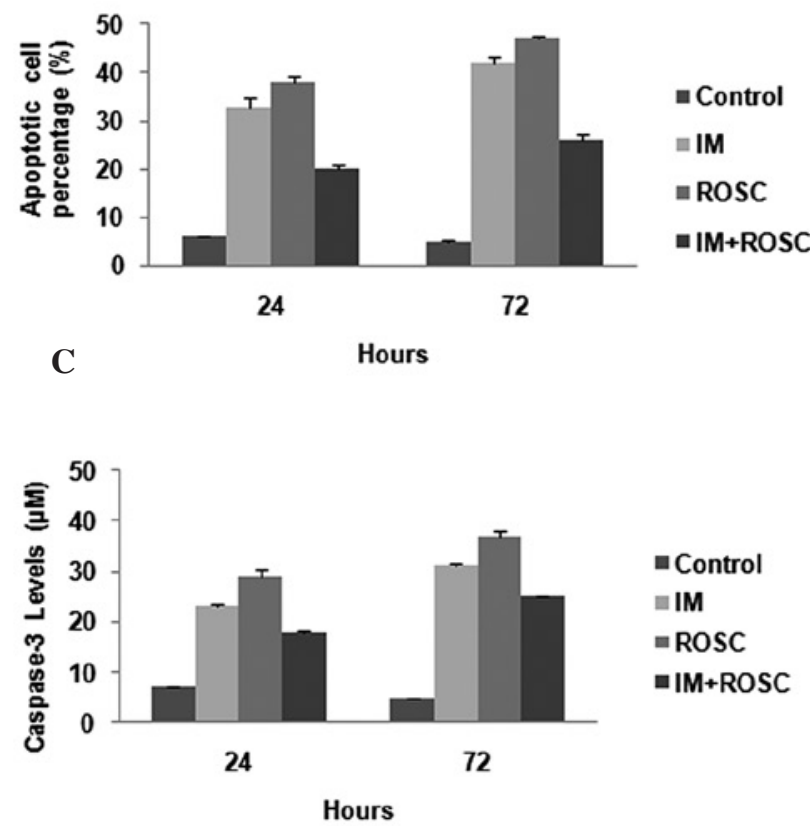

- Control

- IM

$=\mathrm{ROSC}$

$=I \mathrm{M}+\mathrm{ROSC}$

Figure 2. Apoptotic index with caspase-3 levels. (A) The panels of flow cytometric analysis of the apoptotic index. According to the kit's instruction manual, quadrants of panels were defined as: the lower left quadrant of density plots for the numbers of viable cells (annexin $\mathrm{V}^{-}$; $\mathrm{PI}^{-}$), the lower right quadrant for the numbers of earlier stages of apoptotic cells (annexin $\mathrm{V}^{+}$; $\mathrm{PI}^{-}$), the upper right quadrant for the numbers of late stages of apoptotic cells (annexin $\mathrm{V}^{+}$; $\mathrm{PI}^{+}$), and the upper left quadrant for the numbers of dead cells. (B) The graph of apoptotic index. The percentage of apoptotic cells (lower left and upper left of the panels) were presented in the graph. (C) The graph of caspase-3 levels. IM, imatinib mesylate; ROSC, roscovitine; IM+ROSC, the combination of imatinib mesylate and rescovitine. Results are the mean of three different experiments and are presented as the mean \pm SEM. 


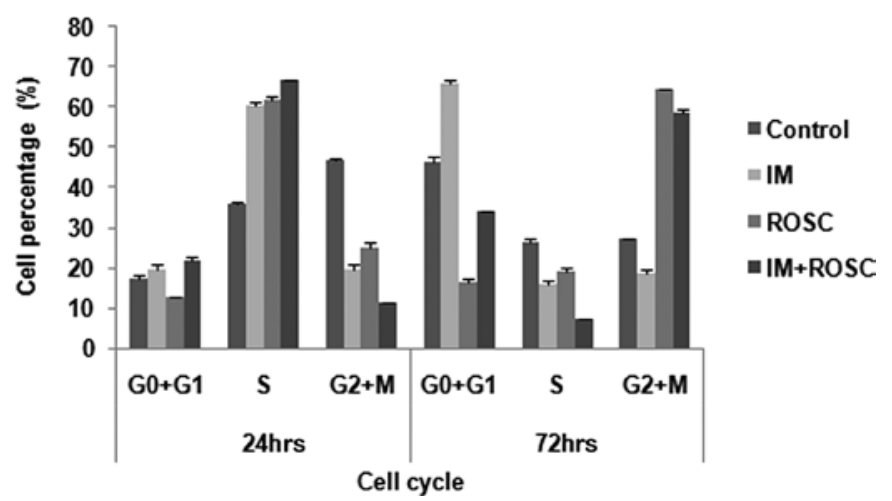

Figure 3. Cell cycle. The percentage of cells at G0/G1-, S- and G2/M-phases are plotted. IM, imatinib mesylate; ROSC, roscovitine; IM+ROSC, the combination of imatinib mesylate and rescovitine. Results are the mean of three different experiments and are presented as the mean \pm SEM.

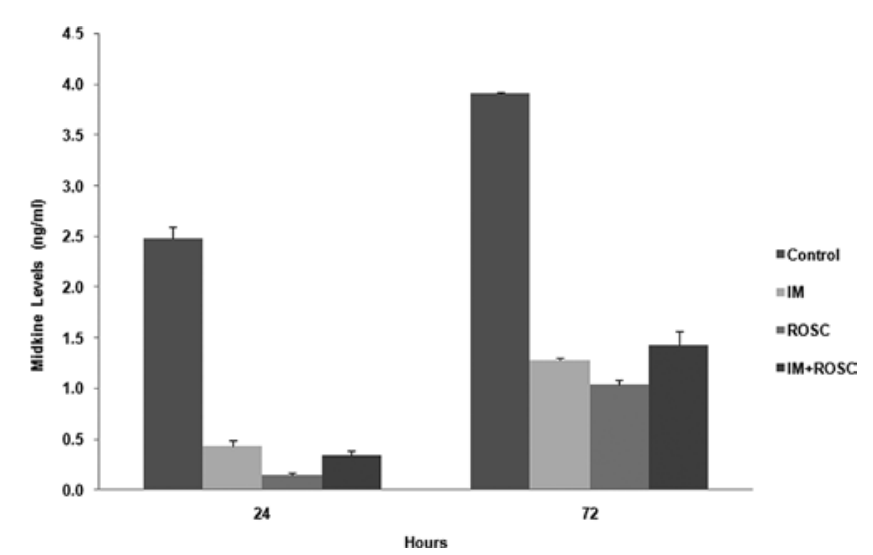

Figure 4. Midkine levels. IM, imatinib mesylate; ROSC, roscovitine IM+ROSC, the combination of imatinib mesylate and rescovitine. Results are the mean of three different experiments and are presented as the mean \pm SEM.

by ROSC ( $>00.05)$. The decrease by IM was much lower than the other groups $(\mathrm{p}<0.05)($ Fig. 5).

EGFR levels. EGFR levels were increased from 24 to $72 \mathrm{~h}$ in the control group cells $(\mathrm{p}<0.05)$ (Fig. 6). All drug applications reduced these levels $(\mathrm{p}<0.05)$. Firstly, ROSC, and secondly, the combination group, reduced EGFR levels much more potently than the well-known tyrosine kinase inhibitor IM $(p<0.05)$.

COX activity. Alterations in COX-1 and COX-2 activities are shown in Fig. 7. The highest decrease in COX-1 levels was determined in the combination group followed by the IM group $(\mathrm{p}<0.05)$. ROSC increased these levels and these increases reached the highest levels between 4th and 7 th min and at the 10th $\min (\mathrm{p}<0.05)$ (Fig. 7A). IM induced the highest decrease in COX-2 activity, and ROSC also decreased this enzyme activity; however, the combination group increased COX-2 activity for $10 \mathrm{~min}(\mathrm{p}<0.05)$ (Fig. 7B).

Protein levels. Alterations in PDGFR- $\alpha$, hTERT, p170, MRP-1 and AQP-4 were evaluated and, results are shown in Fig. 8. IM and the combination group decreased PDGFR- $\alpha$ levels, but ROSC increased these levels $(\mathrm{p}<0.05)$. The highest

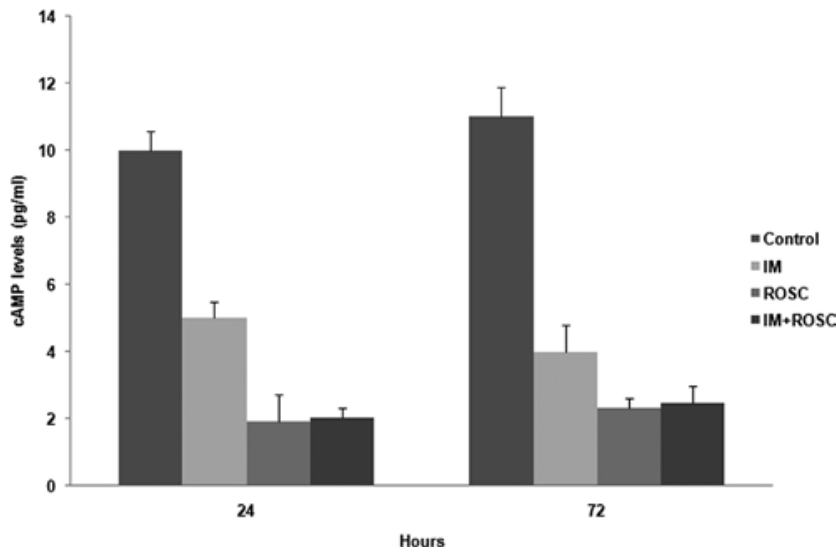

Figure 5. cAMP levels. IM, imatinib mesylate; ROSC, roscovitine; IM+ROSC, the combination of imatinib mesylate and rescovitine. Results are the mean of three different experiments and are presented as the mean \pm SEM.

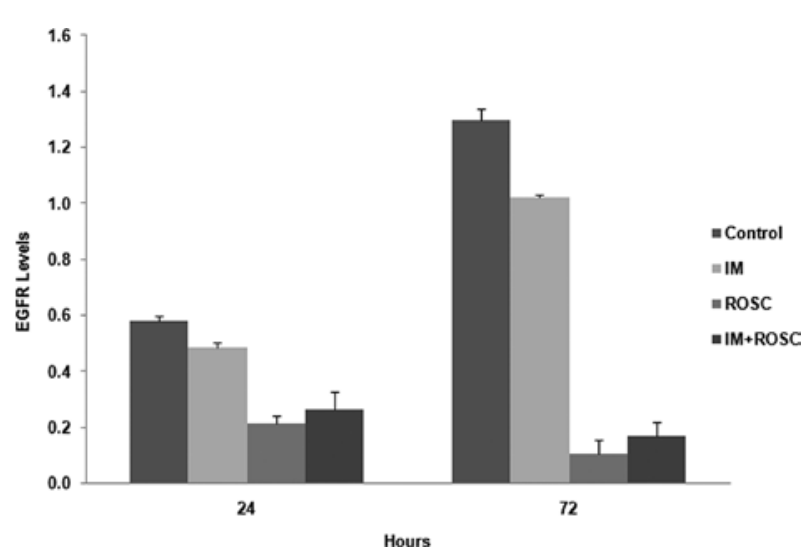

Figure 6. EGFR levels. IM, imatinib mesylate; ROSC, roscovitine; IM+ROSC, the combination of imatinib mesylate and rescovitine. Results are the mean of three different experiments and are presented as the mean \pm SEM.

decrease in PDGFR- $\alpha$ levels was induced by IM ( $\mathrm{p}<0.05)$. IM, ROSC and the combination group increased TERT levels for $72 \mathrm{~h}(\mathrm{p}<0.05)$. The increase in TERT levels by IM was higher than that in ROSC $(\mathrm{p}<0.05)$; however, the increase by the combination group was much higher than that in the ROSC and IM groups $(\mathrm{p}<0.05)$. The combination group induced the highest decrease in p170 levels $(\mathrm{p}<0.05)$, followed by the IM group $(\mathrm{p}<0.05)$. All drug applications decreased MRP-1 levels $(p<0.05)$, but the highest decrease was determined in the combination group, followed by the IM group $(\mathrm{p}<0.05)$. IM decreased AQP-4 levels; however, the combination group and the ROSC group increased AQP-4 levels in T98G GBL cells. This increase was higher in the combination group.

Ultrastructure. Alterations in ultrastructure by TEM are shown in Fig. 9. The control group showed normal morphology characterized by fine-textured nuclear chromatin, intact nuclear membranes and intact cytoplasmic membranes with numerous microvilli, which were in contact with other cells. A number of mitotic cells were observed (Fig. 9A). The IM group showed that cell-to-cell interactions were lost, and eventually small gaps existed between cells. This treatment group also exhibited severe mitochondrial damage, lytic changes 

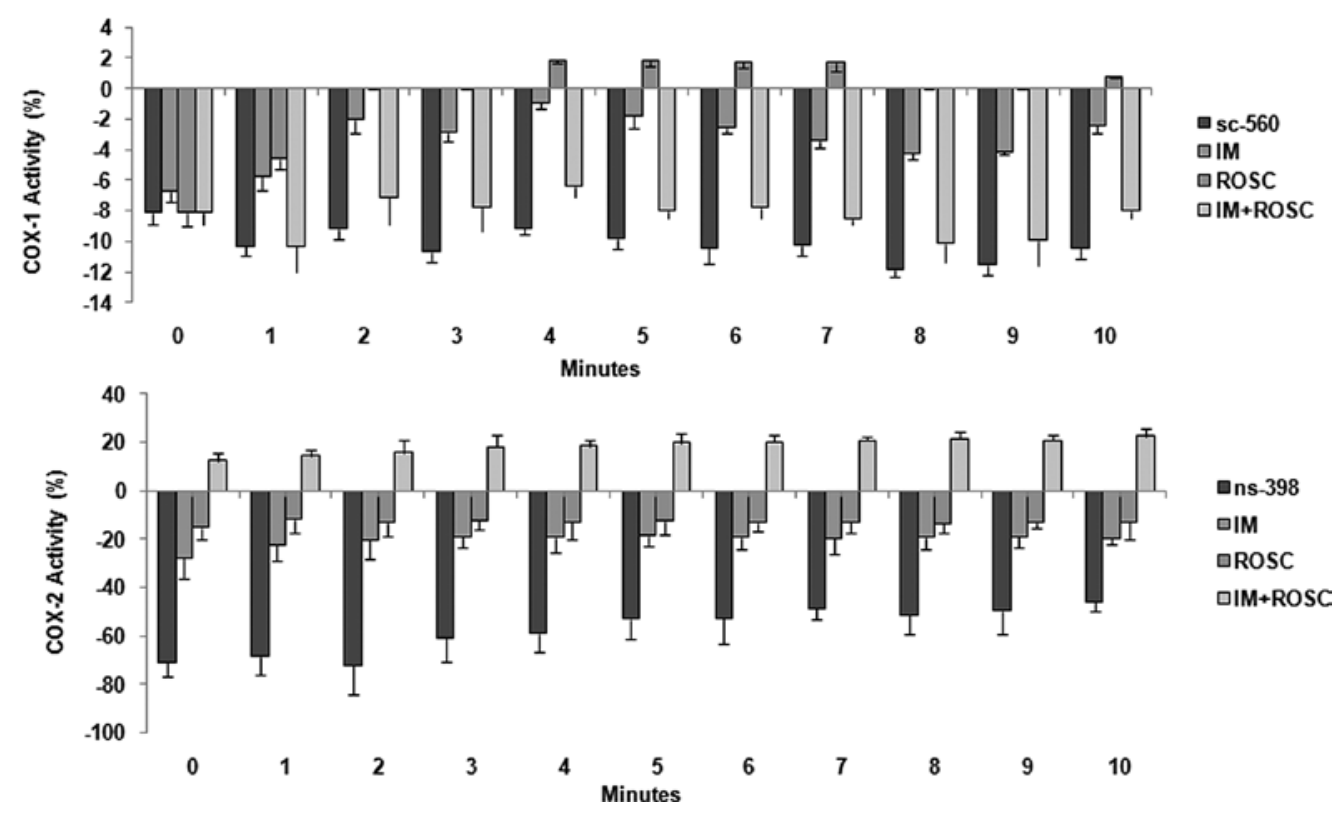

Figure 7. COX-1 and COX-2 levels. sc-560 and ns-398 were selective inhibitors for COX-1 and COX-2, respectively, and were used as positive controls. IM, imatinib mesylate; ROSC, roscovitine; IM+ROSC, the combination of imatinib mesylate and rescovitine. Results are the mean of three different experiments and are presented as the mean \pm SEM.

C IM ROSC IM+ROS

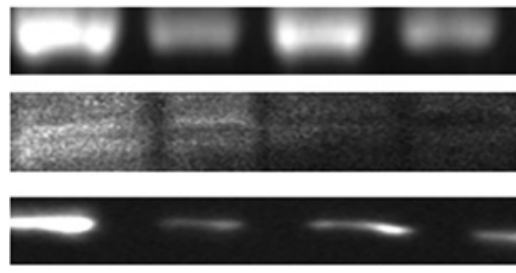

PDGFR- $\alpha$

P-gp

MRP-1

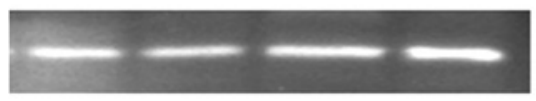

hTERT

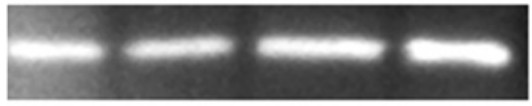

AQP-4

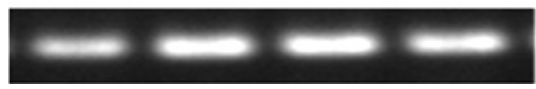

$\beta$-actin

Figure 8. Western blotting analysis of apoptotic and anti-apoptotic protein levels at 72 h. MRP-1 (MW: $190 \mathrm{kDa}$ ), p170 (MW: $170 \mathrm{kDa}$ ), AQP-4 (MW: $34 \mathrm{kDa}$ ), PDGFR- $\alpha$ (MW: $170 \mathrm{kDa}$ ), hTERT (MW: $120 \mathrm{kDa}$ ). IM, imatinib mesylate; ROSC, roscovitine; IM+ROSC, the combination of imatinib mesylate and rescovitine. Results are the mean of three different experiments and are presented as the mean $(\%) \pm$ SEM. Mean values for each group were calculated as the ratio of the density of the sample band to that of the $\beta$-actin band in each lane, multipled by 100 and they are the mean of three different experiments.

such as foamy-vacuolated cytoplasm, and numerous, presumably autophagic, vacuoles. An apoptotic appearance was determined in a number of cells (Fig. 9B). Within the ROSC group, an apoptotic appearance was frequently observed. Spheroids lost their integrity as a result of loss of cell-to-cell interactions and lysis of the cells. Numerous cell remnants in the intercellular area, loss of cell membrane, severe lytic changes, foamy-vacuolated cytoplasm, lipid droplets and few severe mitochondria damage were determined. Notably, the most marked changes in the cytoplasm were the increase in endoplasmic reticulum and ribosomes. A few autophagic vacuoles were also observed in some cells at the time the surrounding endoplasmic reticulum was analyzed (Fig. 9C). In the combination group, little gaps between cells were observed. Vacuoles and vacuole fusions were observed in the cytoplasm. The number of lipid droplets was much higher than that in the ROSC group, and no autophagic vacuoles were determined. Little mitochondrial damage was observed. Notably, as observed in the ROSC group, endoplasmic reticulum and ribosomes were also increased in this group, but the increase in the ribosomes was lower than that in the ROSC group (Fig. 9D). In the ROSC and combination group, mitochondrial damage was often observed (Fig. 9C and D).

\section{Discussion}

ROSC success was confirmed in the treatment of a number of cancer types, including GBL $(1,27)$. In the present study, the combination of ROSC with IM showed an antagonist effect in monolayer and 3D cultures of the human T98G GBL cell line. This antagonism was determined by the highest cell number and the lowest apoptotic index with caspase-3 levels; however, the results provided for the anti-apoptotic protein levels were controversial. The potentiation of TRAIL-induced apoptosis through the downregulation of two major caspase inhibitors, survivin and XIAP, was shown by Kim et al in U87 and T98G GBL cells (5). Kim et al showed that treatment with ROSC recovered the TRAIL-induced activation of caspases in an efficient manner in these cells. In the present study, singly applied ROSC induced the highest increase in caspase-3 levels, but these levels were lowest in the combination group. It seemed that IM blocked the caspase-3 recovery activity of ROSC.

Fleming et al showed that ROSC decreased EGFR levels, and the combination of a new generation receptor tyrosine 

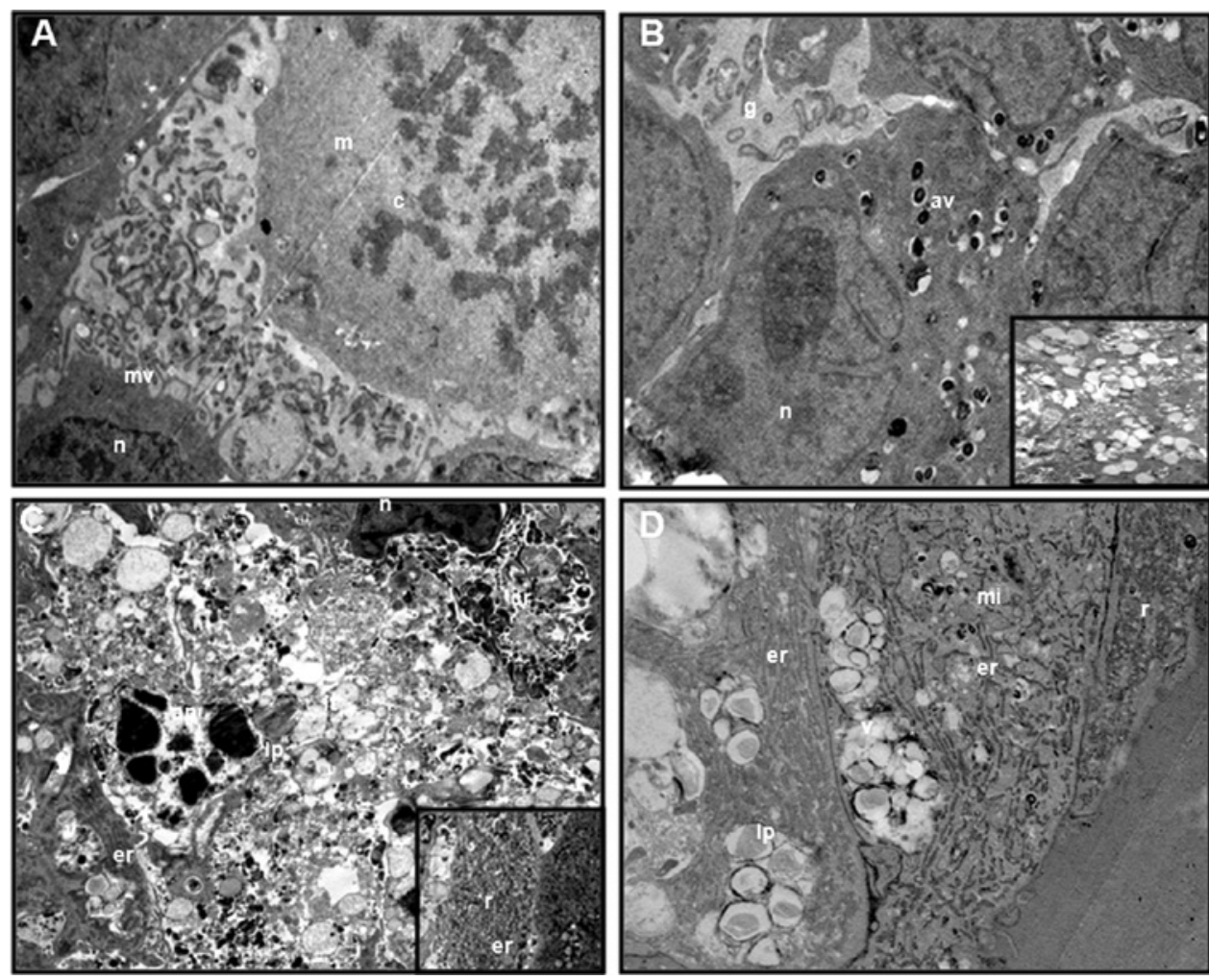

Figure 9. Transmission electron microscopic views of T98G GBM spheroids at $72 \mathrm{~h}$. (A) The control group (original magnification, x10,000). (B) The IM group (original magnification, x10,000 and x7500). (C) The ROSC group (original magnification, $x 7500$ ). (D) The combination group (original magnification, x6000). m, mitotic cell; c, chromosome; mv, microvillus; g, gaps; n, nucleus; av, autophagic vacuole; v, vacuole; lt, lytic cytoplasm; ap, apoptotic cell; er, endoplasmic reticulum; lp, lipid vacuole; r, ribosome; cr, cell remnant; mi, mitochondria.

kinase inhibitor named erlotinib, which acts on EGFR, with ROSC showed a synergistic effect in NSCLC cell lines (28). In agreement with their results, we determined that ROSC and the combination group decreased EGFR levels, respectively, but in contrast to the results by Fleming et al, our combination showed an antagonist effect. Mohapatra et al showed that ROSC reduced the abundance of tyrosine-phosphorylated PDGFR- $\alpha$ receptors in the HTLV-1-transformed T-cell line MT-2 (29). Notably, ROSC increased PDGFR- $\alpha$ levels; however, the combination group decreased PDGFR- $\alpha$ levels.

IM was shown as a substrate for drug efflux proteins, particularly p-gp. This was one of the reasons leading to the failure of IM in GBL treatment (14-16). Spiegl-Kreinecker et al found that a considerable expression of $\mathrm{p}$-gp was relatively rare in glioma cells, in contrast to MRP-1, which was constitutively overexpressed in cells derived from astrocytomas, as well as GBLs such as T98G, and SW1088 cells (30). Komina et al showed that ROSC is capable of inducing apoptosis in the doxorubicin-resistant multiple myeloma cells overexpressing $\mathrm{p}$-gp (6). Consequently, we investigated alterations in these two protein levels. The combination group induced the highest decrease, followed by the IM group. ROSC alone was not as efficient as the others at decreasing these two protein levels. According to our PubMed research, this is the first report to show the inhibitory effect of ROSC on MRP-1 levels. Ding et al reported that AQP-4 is a key molecule involved in maintaining water and ion homeostasis in the central nervous system. In addition, these authors mentioned that AQP-4 is increased in GBL and plays a significant role in
GBL cell migration and invasion, in addition to its well-known function in brain edema (31). The inhibitory effect of ROSC on AQP-2 was shown in kidneys (32). In the present study, IM decreased AQP-4 levels; however, the combination group and ROSC increased AQP-4 levels in T98G GBL cells. This increase was higher in the combination group. There is no report about the effect of ROSC on AQP-4.

Telomeres and telomerase play essential roles in the regulation of the lifespan of human cells. Normal human somatic cells do not, or only transiently, express telomerase and therefore shorten their telomeres with each cell division (33). GBL cancer cells typically express high levels of telomerase and show uncontrolled cell proliferation/growth. Thus, a high telomerase expression allows GBL cells to proliferate, grow and acquire resistance (34). In light of the crucial role of telomerase activity, we also investigated the effects of drugs on TERT protein levels, which is a catalytic subunit of telomerase and required for telomerase activity. Uziel et al reported that IM downregulates telomerase activity and inhibits cell proliferation in telomerase-expressing cell lines such as c-kit-expressing SK-N-MC (Ewing sarcoma), SK-MEL-28 (melanoma), RPMI-8226 (myeloma), MCF-7 (breast cancer) and HSC 536/N (Fanconi anaemia) cells, as well as in ba/F3 (murine pro-B) cells, which do not express c-kit, BCR-ABL or PDGF-R (35). Deville et al showed that hTERT overexpression favors the development of IM resistance through its anti-apoptotic and telomere maintenance functions in CML cells (36). We showed that IM decreased hTERT protein levels slightly; however, ROSC and the combination group increased 
these levels. The combination group led to the highest increase in hTERT protein level. However, as yet we have found no reports on the effect of ROSC on hTERT protein levels.

Cyclooxygenase-1 (COX-1) is constitutively expressed in a wide variety of tissues, whereas the COX-2 gene is highly inducible and expressed in response to stimuli from various cytokines, growth factors and tumor promoters. COX-2 plays a key role in the regulation of progression, invasiveness and angiogenesis of various types of cancer, including gliomas (37). It was shown that COX-2 is upregulated in high-grade gliomas and that COX-2 expression is associated with poor prognosis. Consequently, COX-2 is an emerging target for anti-GBM therapy (38). Studies have shown that ROSC inhibited COX-2 expression in cumulus oocyte complexes and in isolated peritoneal macrophages $(39,40)$, but there is no report about the mechanism of action on COX-1. We showed that ROSC increased COX-1 levels, and that the increased rates reached the highest levels after 4-7 mins and at $10 \mathrm{~min}$. In contrast to ROSC, the combination group induced the highest decrease in COX-1 levels for $10 \mathrm{~min}$. Notably, ROSC and the combination group showed an opposite effect on COX-2 activity; ROSC decreased COX-2 while the combination group increased COX-2. Arunasree et al showed that IM resistance was correlated to high COX-2 activity in IM-resistant K562 cells (41). In the present study, IM was the second drug to inhibit COX-1 and COX-2 activities.

Burger et al showed that ROSC inhibited ribosomal RNA synthesis at early rRNA processing (3). Although this inhibitory effect was reported, notably in the ultrastructure evaluation of ROSC, an increase in ribosomes and endoplasmic reticulum was determined. Few autophagic vacuoles were observed in the ROSC group; thus, autophagy is not involved in the mechanism of ROSC's action as either cell resistance or cytotoxicity. In contrast to our results, Lambert etal determined that ROSC induced autophagy in osteosarcoma cells (42). We showed the correlation between autophagy and the cytotoxicity of IMs in rat glioma cells (14) and in human GBL cells (unpublished data). In the combination group there were no autophagic vacuoles, however, an increase in ribosomes and endoplasmic reticulum was also observed, but this was lower than in the ROSC group alone.

In addition to drug efflux proteins, telomerase and TKR proteins, we also investigated the role of MK in this experiment. Previous reports have suggested that the MK gene may be involved in multidrug resistance $(43,44)$. In our previous report on neuroblastoma, as well as unpublished data in GBL and endometrium carcinoma, we showed the inhibitory effect of IM on MK levels (45). ROSC also had an inhibitory effect on MK levels. However, we did not find any reports about the effect of ROSC on MK. In the combination group, we also determined an inhibitory effect; however, this effect was much weaker than ROSC and IM alone. Dai et al reported that MK played a significant role in rRNA transcription, ribosome biogenesis and cell proliferation in HepG2 cells; thus, low MK levels led to a decrease in cell numbers and ribosome biogenesis (46). In contrast to Dai et al, in the present study, ROSC led to low cell numbers and MK levels, but it also led to an increase in ribosomes. In the report of Riggelen et al, it was mentioned that in certain cases the overexpression of a ribosomal protein has been shown to suppress tumorigenesis
(47). The overexpression of RPL11 has been shown to interfere with cell cycle progression by inhibiting MDM2 (an E3 ubiquitin ligase that targets p53 for degradation), thereby resulting in activation of the p53 pathway (48-51). In light of this study, this ribosome increase may be part of ROSCs. However, in the combination group, the low increase in the ribosome number may reflect antagonism.

In the IM group, severe mitochondrial damage was frequently observed. The decrease in the cAMP level may be due to mitochondrial damage and/or the inhibition of adenylate cyclase. Since the increase in ribosome number requires energy (52), we determined little severe mitochondrial damage in the ROSC and combination groups. Consequently, it may be reported that ROSC decreased cAMP levels through the direct inhibition of adenylate cyclase (AC) activity and ATP was conserved for ribosome biogenesis.

In conclusion, IM with ROSC showed an antagonist effect in the treatment of human GBL cells. The ROSC group was the most efficient group to decrease the cell number and to increase the apoptotic index; however, data provided for the underlying mechanism of its action were controversial, due to the increase in the levels of anti-apoptotic proteins, enzymes and processes (PDGFR- $\alpha$, AQP-4, hTERT, the activity of COX-1 and ribogenesis). The effects of ROSC on the hTERT, MK, AQP-4, MRP-1 levels and COX-1 activity were reported for the first time in the present study. Autophagy was not correlated to ROSC activity in human GBL spheroids. Although the combination group decreased the levels of PDGFR- $\alpha$, EGFR, MK, cAMP, drug efflux proteins and COX-1 activity, it led to the highest increase in hTERT, COX-2 activity and the lowest increase in AQP-4 levels. The ribosome number and the lowest caspase-3 levels seemed to be responsible for one part of this antagonism. GBL MK is not involved in this antagonism. Further investigation is required to identify the key regulatory components which are responsible for this antagonism; however, the determination of this combination therapy as a failure were precautionary for oncologists in the treatment of GBL patients and potentially may contribute to the efficacy of new therapeutic regimens.

\section{Acknowledgements}

This work was supported by the Scientific Research Projects Coordination Unit of Istanbul University (Project number: T988/06102006).

\section{References}

1. Krystof V and Uldrijan S: Cyclin-dependent kinase inhibitors as anticancer drugs. Curr Drug Targets 11: 291-302, 2010.

2. Aldoss IT, Tashi T and Ganti AK: Seliciclib in malignancies. Expert Opin Investig Drugs 18: 1957-1965, 2009.

3. Burger K, Mühl B, Harasim T, et al: Chemotherapeutic drugs inhibit ribosome biogenesis at various levels. J Biol Chem 285: 12416-12425, 2010.

4. Bach S,KnockaertM,Reinhardt J, etal:Roscovitine targets, protein kinases and pyridoxal kinase. J Biol Chem 280: 31208-31219, 2005.

5. Kim EH, Kim SU, Shin DY, et al: Roscovitine sensitizes glioma cells to TRAIL-mediated apoptosis by downregulation of survivin and XIAP. Oncogene 23: 446-456, 2004.

6. Komina O, Nosske E, Maurer M, et al: Roscovitine, a small molecule CDK inhibitor induces apoptosis in multidrug-resistant human multiple myeloma cells. J Exp Ther Oncol 9: 27-35, 2011. 
7. Dey A, Wong ET, Cheok CF, et al: R-Roscovitine simultaneously targets both the p53 and NF-kappaB pathways and causes potentiation of apoptosis:implications in cancer therapy. Cell Death Differ 15: 263-273, 2008

8. Maggiorella L, Aubel C, Haton C, et al: Cooperative effect of roscovitine and irradiation targets angiogenesis and induces vascular destabilization in human breast carcinoma. Cell Prolif 42: 38-48, 2009.

9. Ganapathi SB, Kester M and Elmslie KS: State-dependent block of HERG potassium channels by R-roscovitine: implications for cancer therapy. Am J Physiol Cell Physiol 296: C701-C710, 2009.

10. Yang SY, Miah A, Pabari A, et al: Growth Factors and their receptors in cancer metastases. Front Biosci 16: 531-538, 2011.

11. Natoli C, Perrucci B, Perrotti F, et al: Consorzio Interuniversitario Nazionale per Bio-Oncologia (CINBO). Tyrosine kinase inhibitors. Curr Cancer Drug Targets 10: 462-483, 2010.

12. Waller CF: Imatinib mesylate. Recent Results Cancer Res 184: 3-20, 2010.

13. Razis E, Selviaridis P, Labropoulos S, et al: Phase II study of neoadjuvant imatinib in glioblastoma: evaluation of clinica and molecular effects of the treatment. Clin Cancer Res 15: 6258-6266, 2009.

14. Erguven M, Yazihan N, Aktas E, et al: Carvedilol in glioma treatment alone and with imatinib in vitro. Int $\mathrm{J}$ Oncol 36 : $857-866,2010$

15. Mangiola A, Anile C, Pompucci A, et al: Glioblastoma therapy: going beyond Hercules Columns. Expert Rev Neurother 10: 507-514, 2010.

16. Declèves X, Bihorel S, Debray $\mathrm{M}$, et al: $\mathrm{ABC}$ transporters and the accumulation of imatinib and its active metabolite CGP74588 in rat C6 glioma cells. Pharmacol Res 57: 214-222, 2008.

17. Bihorel S, Camenisch G, Lemaire M, et al: Influence of breast cancer resistance protein (Abcg2) and p-glycoprotein (Abcb1a) on the transport of imatinib mesylate (Gleevec) across the mouse blood-brain barrier. J Neurochem 102: 1749-1757, 2007.

18. Ozawa T, Brennan CW, Wang L, et al: PDGFRA gene rearrangements are frequent genetic events in PDGFRA-amplified glioblastomas. Genes Dev 24: 2205-2218, 2010.

19. Muramatsu T: Midkine (MK), the product of a retinoic acid responsive gene, and pleiotrophin constitute a new protein family regulating growth and differentiation. Int J Dev Biol 37 : $183-188,1993$

20. Garver RI, Chan CS and Milner PG: Reciprocal expression of pleiotrophin and midkine in normal versus malignant lung tissues. Am J Respir Cell Mol Biol 9: 463-466, 1993.

21. Garver RI, Radford DM, Donis-Keller H, et al: Midkine and pleiotrophin expression in normal and malignant breast tissue. Cancer 74: 1584-1590, 1994.

22. Konishi N,Nakamura M, Nakaoka S, et al: Immunohistochemical analysis of midkine expression in human prostate carcinoma. Oncology 57: 253-257, 1999.

23. Mishima K, Asai A, Kadomatsu K, et al: Increased expression of midkine during the progression of human astrocytomas. Neurosci Lett 233: 29-32, 1997.

24. Torres KE, Castillo G and Horwitz SB: Proc Am Assoc Cancer Res 38: 530, 1997.

25. Fan W, Miller MC, Cheng RL, et al: Induction of apoptosis by low concentrations of Taxol is not dependent on a G2/M block. Microsc Microanal 4: 1042-1043, 1998.

26. Miller MC, III, Johnson KR, Willingham MC, et al: Apoptotic cell death induced by baccatin III, a precursor of Taxol, occurs without G2/M arrest. Cancer Chemother Pharmacol 44: 444-452, 1999

27. Yakisich JS, Boethius J, Lindblom IO, et al: Inhibition of DNA synthesis in human gliomas by roscovitine. Neuroreport 10 : 2563-2567, 1999.

28. Fleming IN, Hogben M, Frame S, et al: Synergistic inhibition of ErbB signaling by combined treatment with seliciclib and ErbB-targeting agents. Clin Cancer Res 14: 4326-4335, 2008

29. Mohapatra S, Chu B, Wei S, et al: Roscovitine inhibits STAT5 activity and induces apoptosis in the human leukemia virus type 1-transformed cell line MT-2. Cancer Res 63: 8523-8530, 2003.

30. Spiegl-Kreinecker S, Buchroithner J, Elbling L, et al: Expression and functional activity of the $\mathrm{ABC}$-transporter proteins P-glycoprotein and multidrug-resistance protein 1 in human brain tumor cells and astrocytes. J Neurooncol 57: 27-36, 2002.
31. Ding T, Ma Y, Li W, et al: Role of aquaporin-4 in the regulation of migration and invasion of human glioma cells. Int J Oncol 38: 1521-1531, 2011.

32. Ibraghimov-Beskrovnaya O: Targeting dysregulated cell cycle and apoptosis for polycystic kidney disease therapy. Cell Cycle 6: 776-779, 2007.

33. Shukla S, Acharya S, Rajput D, et al: Telomere-the twilight to immortality. J Assoc Physicians India 58: 553-560, 2010.

34. Marian CO, Cho SK, McEllin BM, et al: The telomerase antagonist, imetelstat, efficiently targets glioblastoma tumorinitiating cells leading to decreased proliferation and tumor growth. Clin Cancer Res 16: 154-163, 2010.

35. Uziel O, Fenig E, Nordenberg J, et al: Imatinib mesylate (Gleevec) downregulates telomerase activity and inhibits proliferation in telomerase-expressing cell lines. $\mathrm{Br} \mathrm{J}$ Cancer 92: 1881-1891, 2005

36. Deville L, Hillion J, Pendino F, et al: hTERT promotes imatinib resistance in chronic myeloid leukemia cells: therapeutic implications. Mol Cancer Ther 10: 711-719, 2011.

37. Greenhough A, Smartt HJ, Moore AE, et al: The COX-2/PGE2 pathway: key roles in the hallmarks of cancer and adaptation to the tumour microenvironment. Carcinogenesis 30: 377-386, 2009.

38. Reardon DA, Quinn JA, Vredenburgh J, et al: Phase II trial of irinotecan plus celecoxib in adults with recurrent malignant glioma. Cancer 103: 329-38, 2005.

39. Du J, Wei N, Guan T, et al: Inhibition of CDKS by roscovitine suppressed LPS-induced *NO production through inhibiting NFkappaB activation and $\mathrm{BH} 4$ biosynthesis in macrophages. Am J Physiol Cell Physiol 297: C742-9, 2009.

40. Vigneron C, Nuttinck F, Perreau C, et al: Effect of roscovitine, a cdk1 inhibitor, and of the presence of oocyte on bovine cumulus cell expansion and cyclooxygenase-2 expression. Mol Reprod Dev 65: 114-21, 2003.

41. Arunasree KM, Roy KR, Anilkumar K, et al: Imatinib-resistant K562 cells are more sensitive to celecoxib, a selective COX-2 inhibitor: role of COX-2 and MDR-1. Leuk Res 32: 855-864, 2008.

42. Lambert LA, Qiao N, Hunt KK, et al: Autophagy: a novel mechanism of synergistic cytotoxicity between doxorubicin and roscovitine in a sarcoma model. Cancer Res 68: 7966-7974, 2008.

43. Hu R, Yan Y, Li Q, et al: Increased drug efflux along with midkine gene high expression in childhood B-lineage acute lymphoblastic leukemia cells. Int J Hematol 92: 105-110, 2010.

44. Kang HC, Kim IJ, Park JH, et al: Identification of genes with differential expression in acquired drug-resistant gastric cancer cells using high-density oligonucleotide microarrays. Clin Cancer Res 10: 272-284, 2004.

45. Bilir A, Erguven M, Yazihan N, et al: Enhancement of vinorelbine-induced cytotoxicity and apoptosis by clomipramine and lithium chloride in human neuroblastoma cancer cell line SH-SY5Y. J Neurooncol 100: 385-395, 2010.

46. Dai LC, Shao JZ, Min LS, et al: Midkine accumulated in nucleolus of HepG2 cells involved in rRNA transcription. World J Gastroenterol 14: 6249-6253, 2008

47. Van Riggelen J, Yetil A and Felsher DW: MYC as a regulator of ribosome biogenesis and protein synthesis. Nat Rev Cancer 10: 301-309, 2011.

48. Dai MS, Arnold H, Sun XX, et al: Inhibition of c-Myc activity by ribosomal protein L11. EMBO J 26: 3332-3345, 2007.

49. Lohrum MA, Ludwig RL, Kubbutat MH, et al: Regulation of HDM2 activity by the ribosomal protein L11. Cancer Cell 3: 577-587, 2003

50. Zhang Y, Wolf GW, Bhat K, et al: Ribosomal protein L11 negatively regulates oncoprotein MDM2 and mediates a p53dependent ribosomal-stress checkpoint pathway. Mol Cell Biol 23: 8902-8912, 2003

51. Bhat KP, Itahana K, Jin A, et al: Essential role of ribosomal protein L11 in mediating growth inhibition-induced p53 activation. EMBO J 23: 2402-2412, 2004.

52. Muller AW: Thermosynthesis as energy source for the RNA World: a model for the bioenergetics of the origin of life. Biosystems 82: 93-102, 2005. 